\title{
Multi-Objective Re-entry Trajectory Optimization based on the Physical Programming Method for Hypersonic Gliding Vehicle
}

\author{
Haiyan Chu, Jian Li \\ National University of Defense Technology, Changsha 410073, China \\ Xiaoyanzi905@qq.com
} Keywords: Physical programming method, multi-objective optimization, re-entry trajectory
optimization, Gauss pseudo spectral method, hypersonic gliding vehicle.

\begin{abstract}
Re-entry trajectory optimization is a key step in the scheme planning of the hypersonic gliding vehicle. The problem of multi-objective re-entry trajectory optimization is usually solved by the weighted summary method (WSM). However, it is difficult to obtain the non-convex Pareto noninferior solution. To overcome this problem, the trajectory optimization is established with the physical programming (PP) method, which is capable to obtain solution with less computational intensity and reflect the designer's preference. Taking a hypersonic gliding vehicle as a numerical example, the numerical simulation considers five objectives: maximum terminal velocity, maximum range, minimum peak value of heating rate, and minimum oscillation. In addition, Gauss pseudo spectral method can accurately satisfy variable constraints. Performance of PP-based re-entry trajectory optimization is demonstrated in 3-D nonlinear dynamics scenario. The numerical simulation results show that it can easily realize and improved the performance of the hypersonic gliding vehicle. The research has certain engineering application value.
\end{abstract}

\section{Introduction}

In the recent decades, hypersonic vehicles have been drawing a lot of attention, and it will be the most suitable weapon to conduct prompt global strike in the future. In order to improve the survivability and fighting strength of hypersonic vehicles, it is significant to consider different objectives into the re-entry trajectory optimization.

Additionally, the weighted summary method (WSM) is widely adopted in the multi-objective trajectory optimization. Chen Gang ET a1.solved the multidisciplinary optimization of re-entry trajectory with weighting processing, the genetic algorithm and the sequential quadratic programming method [1]. Seereeram ET a1.achieved the target spacecraft trajectory optimization using WSM and genetic algorithm [2]. Thus, the weights in WSM makes the selection of optimization algorithm is very flexible. However, it is difficult to determine the subjective weights. Determining a group weights to meet the need of users usually costs most of the time.

Meanwhile, an effective and computationally efficient approach, the physical programming, was proposed by Messac in 1995 [3]. Compared with traditional WSM, PP method have many advantages in multi-objective trajectory optimization. It can obtain the reliable optimization results with minimal prior knowledge.PP method has been developed by many scholars and has been applied increasingly in variable areas. In [4], the multi-objective re-entry trajectory optimization was realized by the genetic algorithm and PP method. It demonstrates the efficiency of PP method. However, it did not consider the no-fly zone, this means that it needs to consider more actual situation.

In this paper, taking a hypersonic gliding vehicle as numerical example, the numerical simulation considers five objectives and multiple constraints into the process of re-entry trajectory optimization. Furthermore, the model is established with PP method, and the standard optimization model with one comprehensive objective and multiple constraints is solved by Gauss pseudo spectral method, which has good stability and robustness.

The rest of this paper is organized as follows. The mathematic model is presented in the next section. The theory of PP method is given in Sec 3. The simulation results and the analysis of the reentry process are then drawn in Sec 4. Finally, the appropriate conclusions are given in Sec 5. 


\section{Mathematic Model for Hypersonic Gliding Vehicles}

\subsection{Dynamics Model Description of Gliding Vehicles}

In this section, the dynamic model utilized in the three-dimensional re-entry guidance problem is presented [5]. The nonlinear equations over a spherical, rotating Earth can be described as:

$$
\begin{gathered}
\dot{r}=V \sin \gamma \\
\dot{\theta}=-\frac{V \cos \gamma \sin \psi}{r \cos \phi} \\
\dot{\phi}=\frac{V \cos \gamma \cos \psi}{r} \\
\dot{V}=-D-g \sin \gamma+\omega^{2} r \cos \phi(\sin \gamma \cos \phi-\cos \gamma \sin \phi \cos \psi) \\
\dot{\gamma}=\frac{1}{V}\left[\mathrm{~L} \cos \sigma+\left(\frac{V^{2}}{r}-g\right) \cos \gamma\right]+2 \omega \cos \phi \sin \psi+\frac{\omega^{2} r}{V} \cos \phi(\cos \gamma \cos \phi-\sin \gamma \sin \phi \cos \psi) \\
\dot{\psi}=-\frac{\mathrm{L} \sin \sigma}{V \cos \gamma}+\frac{V}{r} \cos \gamma \sin \psi \tan \phi+2 \omega(\sin \phi-\tan \gamma \cos \phi \cos \psi)+\frac{\omega^{2} r}{V} \cos \gamma \cos \psi \sin \phi \cos \phi
\end{gathered}
$$

Dimensionless aerodynamic drag acceleration and lift acceleration are given by

$$
D=\frac{C_{D} \rho V^{2} S}{2 m} \quad L=\frac{C_{L} \rho V^{2} S}{2 m}
$$

\subsection{Constraints}

During this progress, motion of the vehicles is restricted with the constraints as followed.

(1) Dynamic pressure constraint :

$$
q=0.5 \rho V^{2} g_{0} R_{e} \leq q_{\max }
$$

(2) Heat flux constraint :

$$
\dot{Q}=2^{0.5} \mathrm{Cq}^{0.5} V^{2.15} \leq \dot{Q}_{\max }
$$

(3) Normal load factor constraint :

$$
\backslash N=q S \sqrt{C_{L}^{2}+C_{D}^{2}} / m g_{0} \leq N_{\max } \backslash
$$

(4) Terminal constraints :

$\backslash r_{f}=r_{d}, \theta_{f}=\theta_{d}, \phi_{f}=\phi_{d}, \gamma_{f}=\gamma_{d} \backslash$

(5) Guidance command constraints: The gliding vehicles fly within control variables' upper and lower bounds.

\section{Physical Programming: Effective Optimization for Computational Design}

\subsection{Classification of Design Metrics and Preference Functions}

PP method is an effective method to solve multi-objective optimization problem. It can transform different kinds of objectives into a general objective. The basic idea is to set the preference functions, which reflect the designer's preference for the design objectives. In the process of PP, designers classify preferences as four different types. And each type of preference is divided into hard preferences $(\mathrm{H})$ and soft preference $(\mathrm{S})$. Fig. 1 describes the meaning of each type of preference functions qualitatively. The horizontal axis represents the design measures, and the vertical axis represents design metric value. 


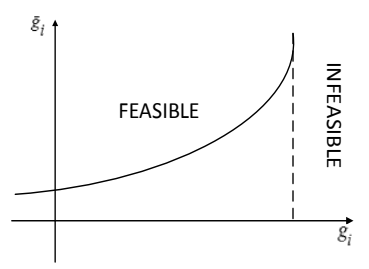

(a) Class 1-S

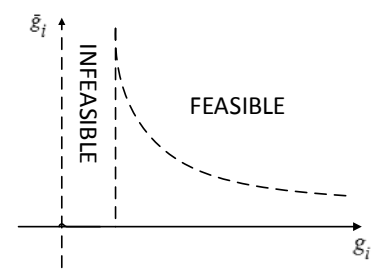

(c) Class 2-S

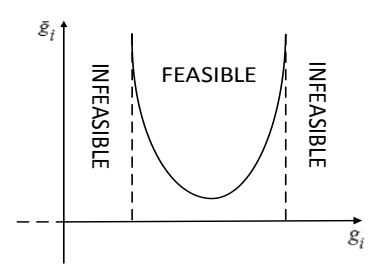

(e) Class 3-S

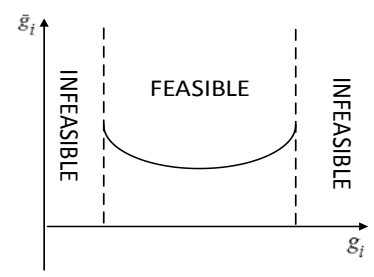

(g) Class 4-S

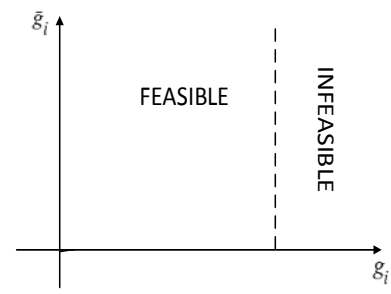

(b) Class 1-H

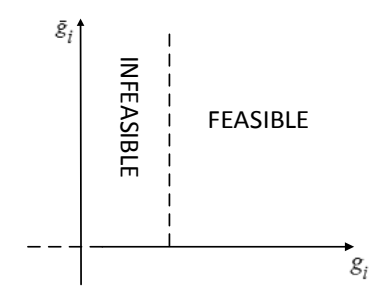

(d) Class 2-H

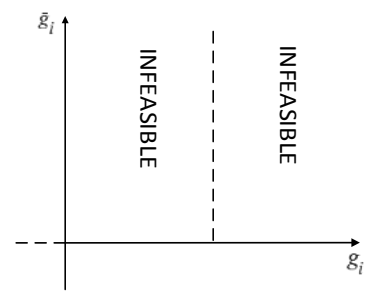

(f) Class 3-H

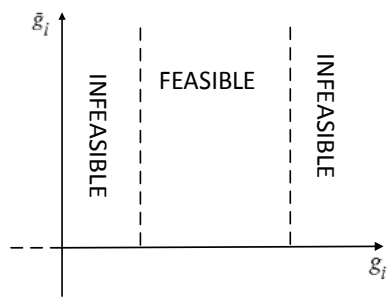

(h) Class 4-H

Fig. 1 Type of Preference Function

\subsection{Mathematical description of the preference function}

According to the different satisfaction degree, the values of preference function are divided into several areas. Taking Class 1-S preference function as an example, we can see the design of the metric in Fig.2:

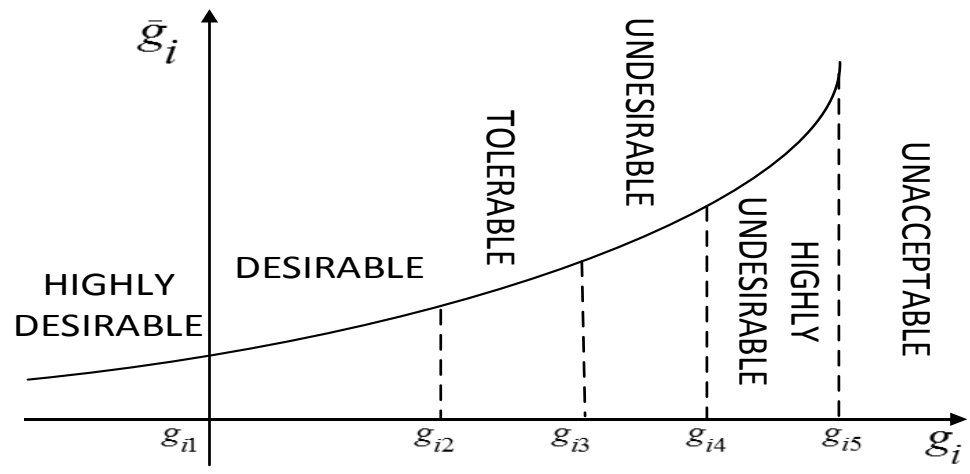

Fig. 2 Interval Division of Class 1-S Preference Function

And then, the solving process of the preference function is as follows:

Step 1: Solve the difference between the regional boundaries.

Step 2: Get the preference value of regional borders.

Step 3: Solve the difference between boundaries.

Step 4: Solve the cutting rate of the boundary.

Step 5: Solve the slope of the boundary.

Step 6: According the following equations, solve the curve parameters in Zone 2 5.

$S_{i-1}=-\Delta x_{i}^{3} b / 3+c \quad g_{P i-1}=\Delta x_{i}^{4} b / 12+d \quad S_{i}=\Delta x_{i}^{3} \mathrm{a} / 3+c \quad g_{P i}=\Delta x_{i}^{4} \mathrm{a} / 12+\Delta x_{i} c+d$

Step 7: If the value is in Zone 1, the preference function can be expressed as

$$
\begin{aligned}
& g_{P}=g_{P 1} e^{S_{1}\left(\mathrm{~g}-\mathrm{g}_{1}\right) / \mathrm{g}_{P 1}} \\
& g_{P}=\Delta x_{i}^{4}\left[\frac{a \bar{x}_{i}^{4}}{12}+\frac{b\left(\overline{\mathrm{x}}_{i}-1\right)^{4}}{12}\right]+c \Delta x_{i} \overline{\mathrm{x}}_{i}+d \quad i=2,3,4,5
\end{aligned}
$$

Step 8: Finally, the target preference function can be written as $g_{P_{T}}=\lg \left(\frac{1}{n_{s c}} \sum_{j=1}^{m} g_{P_{j}}\right)$.

\section{Simulation Results and Analysis}

\subsection{Single Objective Trajectory Optimization}

According to the analysis, the optimization goals in this paper are the maximum range, the maximum terminal vehicle, the minimum oscillation, the minimum peak value of heating rate, and 
the minimum difference between the terminal height and the ideal height. Moreover, the Gauss pseudo spectral method is used to solve the five single objective trajectory optimization problems.

Evidently, we can find the correlation between the different single objective re-entry trajectory optimization. When the trajectory is most stable, the peak value of heating rate is relatively small. Of course, there are also conflicts between different objectives. The trajectory shocks more severe under the objective of farthest range. Generally, there is no ideal design to achieve all objectives. It seems that the improvement of an objective is often based on the poor performance of other objectives.

Analyzing the single objective optimal trajectory can help to determine the preference structure. The preference structures of multi-objective trajectory optimization are designed in Table 1.

Table 1 Preference Structures of Multi-Objective Trajectory Optimization

\begin{tabular}{ccccccc}
\hline \multirow{2}{*}{ Objective } & Preference & \multicolumn{5}{c}{ Value of Boundary } \\
\cline { 3 - 7 }$f_{1} /(\mathrm{km})$ & Function & $g_{i 1}$ & $g_{i 2}$ & $g_{i 3}$ & $g_{i 4}$ & $g_{i 5}$ \\
$f_{2}$ & Class 2-S & 2500 & 2400 & 2300 & 2200 & 2100 \\
$f_{3}$ & Class 2-S & 1.015 & 1.01 & 1 & 0.95 & 0.9 \\
$f_{4} /\left(\mathrm{KW} / \mathrm{m}^{2}\right)$ & Class 1-S & 1.5 & 1.55 & 1.58 & 1.6 & 1.63 \\
$f_{5} /(\mathrm{Km})$ & Class 1-S & 1671.74 & 1671.75 & 1671.76 & 1671.78 & 1671.8 \\
\hline $4.2 \mathrm{~T}$ & Class 1-S & 42 & 45 & 48 & 50 & 52 \\
\hline
\end{tabular}

4.2 The Re-Entry Trajectory Obtained By PP Method

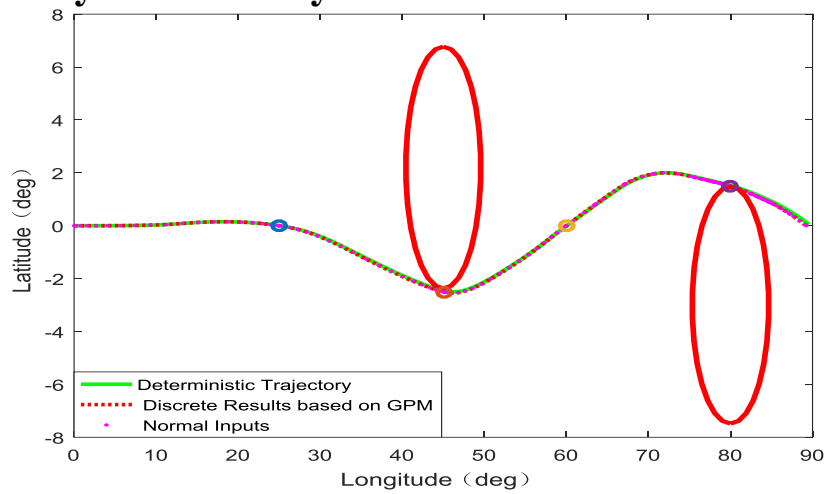

Fig. 3 Flat Trajectory

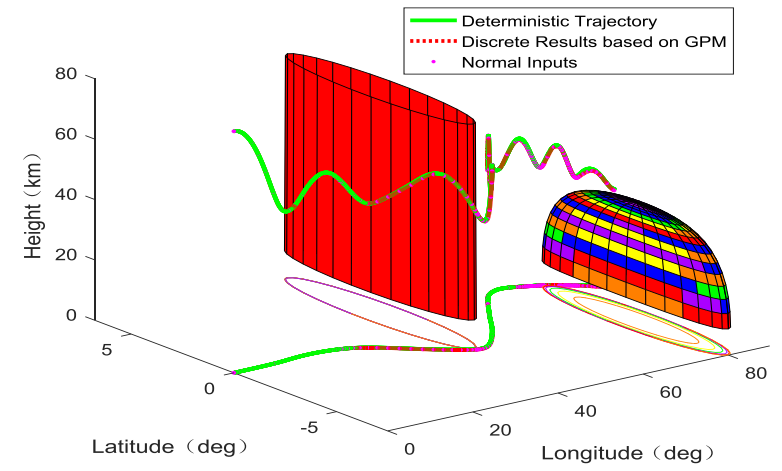

Fig. 4 Three-Dimensional Figure of Re-entry Trajectory

Comparing the re-entry trajectory obtained by PP method with the re-entry trajectory optimized by the single target trajectory, it can be seen that the former can get the optimal solution that satisfies the designer's preferences.

\subsection{PP Method VS WSM}

Table 2 Weights in the WSM Simulation

\begin{tabular}{cccccc}
\hline weight & a1 & a2 & a3 & a4 & a5 \\
\hline First time & 0.3 & 0.1 & 0.25 & 0.25 & 0.1 \\
Second time & 0.1 & 0.15 & 0.3 & 0.3 & 0.15 \\
\hline
\end{tabular}




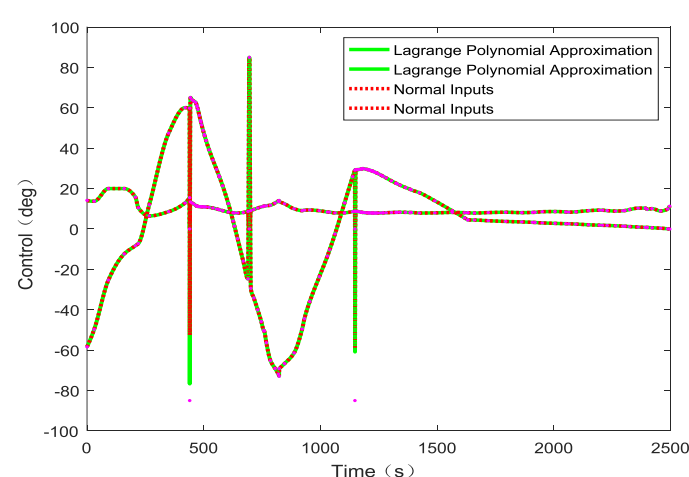

First time

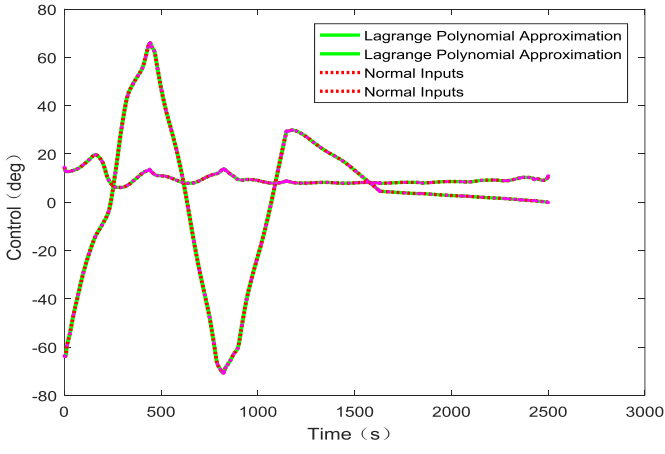

Second time

Fig. 5 Two Situation of Trajectory Obtained by WSM

In Fig 6, the re-entry trajectories obtained by PP method are compared with the re-entry trajectories obtained by WSM. The given flow chart illustrates the process of the trajectory optimization by PP method and WSM respectively. It works as Fig. 7 shows.

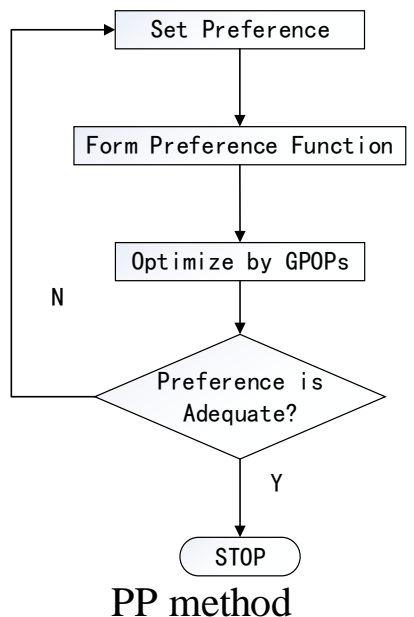

PP method

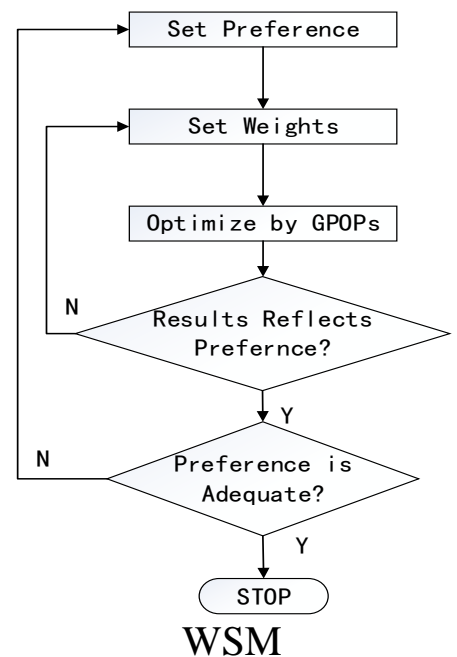

WSM

Fig. 6 the process of the trajectory optimization by PP method (a) and WSM (b)

Although both of them can realize the multiple objective trajectory optimization, WSM requires the designer to give each weight coefficient objectively. Once the result is not ideal, it is necessary to repeatedly set every weight until the results are satisfied. At the same time, the more the quantity of objective is, the more changeable and complicated the weight setting is. However, as long as the designer give the desired target preference value according the actual situation, the efficiency of the design and calculation can be optimized. Therefore, PP method can reduce the time to calculate the multi-objective re-entry optimization trajectory.

\section{Conclusion}

The basic ideas and mathematical modeling process of PP method are introduced. The trajectory optimization problem of the hypersonic gliding vehicle is studied in combination with the Gaussian pseudospectral method. Through the comparison of the optimization results, it is demonstrated that PP method has the advantages over the other methods. Additionally, it is more efficient and robust, and is insensitive to initial value. It could achieve better optimization results. In addition, it has the ability of achieving the non-convex Pareto non-inferior solution. The maximal range, maximum terminal speed, the ballistic stability and minimum peak of the heat flux density are considered. The simulation results demonstrate the advantages of PP method in response to the designer's preference, and the physical causes of the optimal trajectory generation are analyzed. Numerical simulation results validates the high performance about computational efficiency and accuracy and also shows the great robustness with different ranges of perturbation in the aerodynamic conditions. Thus, PP 
method proposed in this article is proved to be quite practical in multi-objective trajectory optimization.

\section{References}

[1]. Chen Gang, Xu Min, Wan Ziming, ET a1.Multidisciplinary design optimization of re-entry trajectory [J]. Journal of Astronautics, 2006, 27 (6): 1147-1150.

[2]. Seereeram L, Ravichandran M, ET a1. Multi-spacecraft trajectory optimization and control using genetic algorithm techniques[C].Proceedings of Aerospace Conference. [s.1.]: IEEE, 2000: 99108.

[3]. Messac A. Physical programming: effective optimization for computational design [J]. AIAA Journal, 1996, 34 (1): 149-158.

[4]. Yong Enmi, Chen Lei, Tang Guojin. Trajectory optimization of hypersonic gliding re-entry vehicle based on the physical programming [J]. ACTA Aeronautica ET Astronautica Sinica, 2008, 29 (5): 1091 1097.

[5]. John H., Robert J. Advanced guidance and control methods for reusable launch vehicles: test results. AIAA Guidance, Navigation, and Control Conference and Exhibit[C].2002. 